\title{
INSCRIPCIÓN ROMANA DEL YACIMIENTO DE GIBALBÍN (CÁDIZ) CON INDICACIÓN DE SU CONDICIÓN DE MUNICIPIO
}

\author{
ROMAN INSCRIPTION FROM THE SITE OF GIBALBÍN (CADIZ) \\ WITH AN INDICATION OF ITS MUNICIPALIS STATUS
}

\author{
JULIÁN GONZÁLEZ FERNÁNDEZ*
}

Resumen: El hallazgo de un fragmento de inscripción en bronce en el yacimiento de Gibalbín, en el que se puede leer MVN.V..., lleva al autor a suponer, de forma conjetural, que el texto de Plinio Vgia cognominata Castrum Iulium, item Caesaris Salutariensisse refiere en realidad no a una ciudad con dos cognomina, sino a dos ciudades diferentes, según el testimonio de Tolomeo (2.4.10). Por último, el autor se inclina por ubicar en Gibalbín a Vgia Castrum Iulium.

Palabras clave: Epigrafía, topografía, geografía, ciudades romanas, Vgia Castrum Iulium, Romanización.

A unos $20 \mathrm{~km}$ al noreste de Jerez de la Frontera se levanta el cerro de Gibalbín, que domina toda la región y en el que existen restos de murallas, de unas termas y otros impresionantes elementos arquitectónicos, cerámica, etc. (Sillières 1977: 342-43, 1990: 437), que revelan la existencia en este lugar de una importante ciudad antigua, cuyo nombre se desconoce, pues los diversos fragmentos de inscripciones encontradas en el mismo (Fita 1896: 432-35, Romero de Torres 1934: 202, González 1982: 111-13), dado su escaso tamaño, no han contribuido a solucionar el problema.

\footnotetext{
* c/ Monsalves, 27, 41001. Correo-e: 72Germanico@gmail.com
}

\begin{abstract}
The discovery of a fragment of inscription on bronze at the site of Gibalbín, in which you can read MVN.V..., leads the author to guess, so conjectural, the text of Plinius Vgia cognominata Castrum Iulium, item Caesaris Salutariensis refers indeed not to a city with two cognomina, but to two different cities, according to the testimony of Tolomeo (2.4.10). Finally, the author is inclined to put in Gibalbín Vgia Castrum Iulium.

Key words: Epigraphy, topography, geography, Roman cities, Vgia Castrum Iulium Romanization.
\end{abstract}

No obstante, no han faltado autores que se han aventurado en suposiciones muy arriesgadas a dar un nombre a la ciudad oculta bajo las ruinas de Gibalbín; así, por ejemplo, Tovar, seguido por Chic, cree que se trataría de Cappa (Tovar 1974: 49, Chic 1979-80: 275-76), ciudad mencionada en la vía Baesippo-Hispalis, entre Ugia y Saguntia (A. Rav., 417: 5-7, Roldán 1975: 133, Sillières 1990: 433-35). También está mencionada en Plinio (N.H. 3,15) entre las ciudades estipendiarias del convento Gaditano. Sillières, que ha seguido detenidamente el trazado de esta vía y estudiado los yacimientos arqueológicos que por su importancia pudieran corresponder a alguna ciudad antigua, sitúa Cappa en el Cerro Esperilla, situado a unos $10 \mathrm{~km}$ al noroeste de la villa de Espera, donde hay un extenso yacimiento ibero-romano de al menos 20 ha, en el que se ven en superficie numerosos 
restos de edificaciones y cisternas realizadas en la roca, así como terra sigillata aretina, galo-romana, hispánica y algo de sigillata clara A, C y D (Sillières 1990: 437).

En 1994 tuve la oportunidad de publicar un fragmento de diploma militar fechado el 17 de abril del año 166 d.C., en el reinado conjunto de Marco Aurelio y Lucio Vero, perteneciente a un pretoriano de la cohors X (González 1994: 11-15). Desgraciadamente no se ha conservado ni el nombre ni la origo del mismo, pero sabemos que las cohortes pretorianas en el momento de su formación se reclutaban exclusivamente en Italia en las ciudades de derecho latino antiguo y en las colonias romanas, según el testimonio de Tácito (Ann. 4,5: Etruria ferme Umbriaque delectae aut vetere Latio et colonias antiquibus Romanis), y en el período anterior a su reorganización realizada por Septimio Severo, además en algunas específicas provincias: Hispania, Macedonia y Norico, según nos cuenta Casio

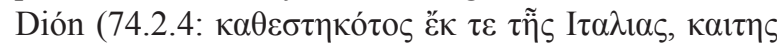

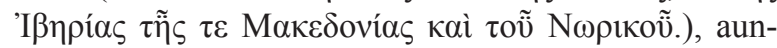
que el primer testimonio provincial que conocemos es de época de Claudio (Passerini 1939: 160). Posteriormente, los acontecimientos que tuvieron lugar los años 68/69 y la política de incorporación de los provinciales a la vida política del Imperio iniciada por Vespasiano contribuyeron sin duda a abrir estas unidades de élite a individuos procedentes de las provincias, aunque siempre de colonias y/o municipios. A pesar de esta progresiva incorporación, conviene constatar que el $86 \%$ de los pretorianos son de origen itálico y tan sólo el 14\% son provinciales (Roldán 1974: 263-264, 328-29).

El hecho de que los soldados, al finalizar su servicio militar, o bien regresaban a su lugar de nacimiento o bien se establecían en la provincia donde habían terminado su vida militar, o, en último caso, en algún lugar próximo (Raepsaet-Charlier 1978: 557-562), y que los pretorianos procedían de ciudades privilegiadas, nos lleva a suponer que este sería el status de la ciudad cuyas ruinas se encuentran en el despoblado de Gibalbín, y lo que es más importante, este status tendría que ser el de una ciudad dotada de Latio vetere o una colonia romana. Como conocemos los nombres de las nueve coloniae civium Romanorum de la Bética, según el testimonio de Plinio, la conclusión es evidente: la ciudad situada en el despoblado de Gibalbín sería una colonia o municipio de derecho latino antiguo, anterior a la concesión del ius Latii por Vespasiano.

Hace algunos años tuve ocasión de examinar y fotografiar en una colección particular de Sevilla un pequeño fragmento de bronce procedente, según me informó su propietario, del yacimiento de Gibalbín, que solo ha conservado su margen inferior y curiosamente uno de los clavos con los que se incrustaba en algún edificio público. Mide $9 \mathrm{~cm}$ de altura, $11 \mathrm{~cm}$ de anchura y $0,5 \mathrm{~cm}$ de grosor; la altura de las letras es de $2,4 \mathrm{~cm}$; el signo de interpunción parece una hedera estilizada que adopta la forma de una flor de lis y tan sólo ha conservado una línea de texto (fig. 1):

$$
\text { [- - - ]MVN.V[- - - ] }
$$

Dos son los datos importantes aportados por este singular fragmento: uno, que el nombre de la desconocida ciudad ubicada en el yacimiento de Gibalbín empezaba por V-, y dos, que el status de la ciudad era el de municipio.

En su relación de ciudades del convento Gaditano, Plinio $(3,1,15)$ nos proporciona dos, cuyos nombres empiezan por $\mathrm{V}$-, una entre las dotadas del derecho latino y una segunda, entre las estipendiarias: Latinorum... Vrgia cognominata Castrum Iulium, item Caesaris Salutariensis; stipendiaria...Vsaepo.

La similitud de grafía de Vsaepo con Saepo, ciudad mencionada por Plinio entre las ciudades de la Beturia Céltica $(3.1,14)$, ha llevado a algunos estudiosos a dudar de la existencia de una de las dos y considerar que se trataba de una sola. Así, por ejemplo, Hübner menciona dos inscripciones (CIL II 1339-1340) encontradas en la Dehesa de la Fantasía (Cortes de la Frontera, Cádiz), en las que, según su parecer, se leía res $p$. V. Saeponensium, con un punto entre la $\mathrm{V}$ y la $\mathrm{S}$, y llega a la conclusión de que la $\mathrm{V}$ correspondería a un cognomen como V(ictrix) y que el Vsaepo de Plinio había de leerse como V(ictrix) Saepo (Hübner, CIL II: p. 180, 846; García 1971: 100, Tovar 1974: 61, Thouvenot, 1940: 199). Detlefsen (1870: 303-04), por el contrario, defendía la identidad de ambas ciudades y localizó Vsaepo en Cortes de la Frontera, en tanto que el emplazamiento de Saepo le resultaba incierto (una opinión semejante han mantenido, entre otros, Albertini 1923: 95, Hoyos 1979: 449, Wiegels 1985: 65). Schulten (RE 1.A 1724; 9.A 1073), que también piensa en ciudades diferentes, ubica, sin embargo, Saepo en la Dehesa de la Fantasía y considera el emplazamiento de Vsaepo desconocido

El hallazgo en la primavera de 1982, en el cortijo Vistalegre, término municipal de Olvera (Cádiz), en un lugar próximo a la finca Orihuela, donde se encontró la inscripción funeraria de M. Clodius Rufinus (González 1982: 223-232, M. Clodi Rufini an. / XXIII [a] b latroni/b. ocisus est s.t.t.), de un espléndido pedestal dedicado a Trajano por la res p(ublica) Saeponensisum 


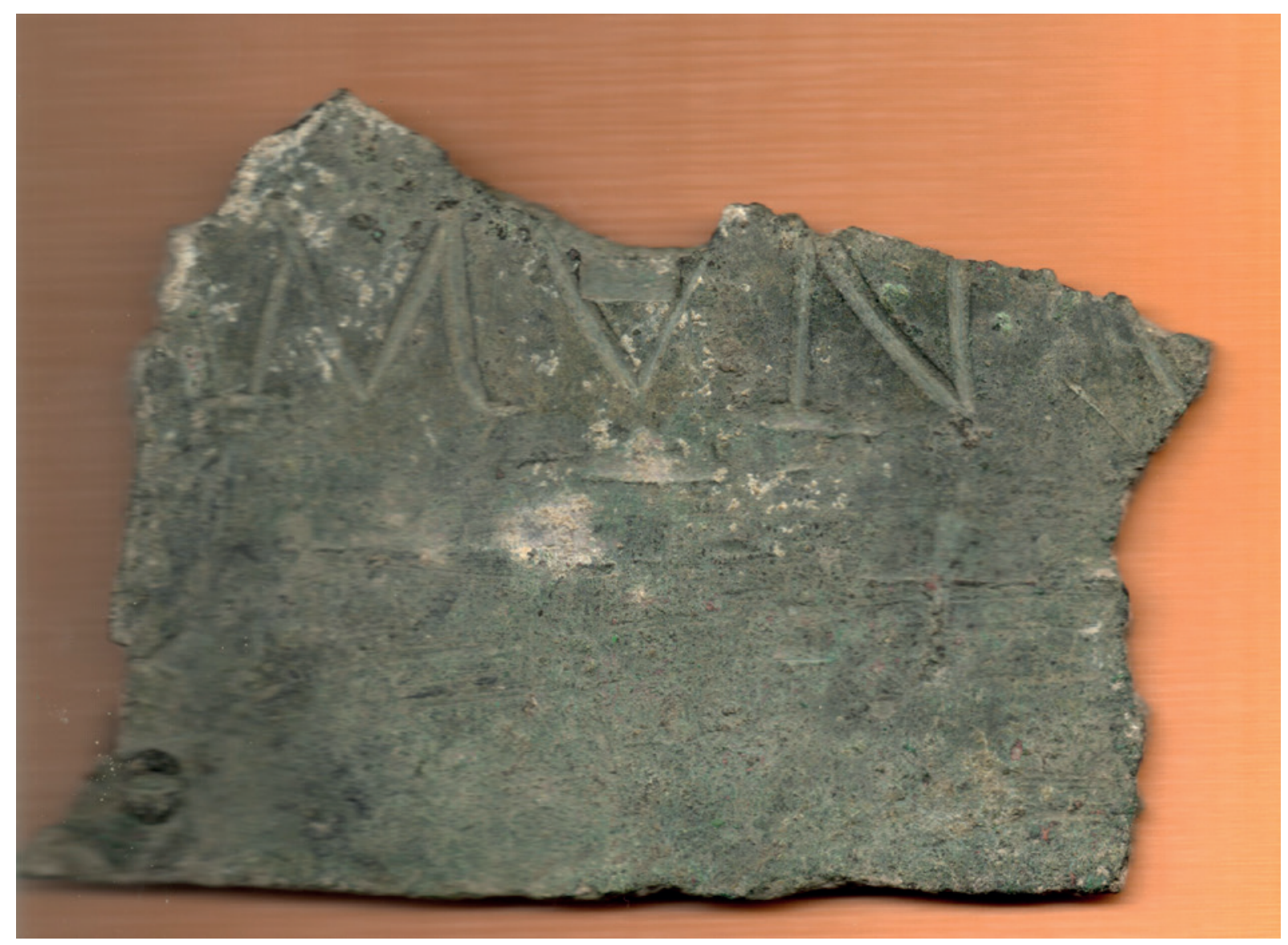

Figura 1.

(González 1987: 238-242, Imp. Caes. D[ivi Ner]/vaef. Ne[rv]ae Traiano / [Optimo A]ug. Ger. Daci/co Part(h) ico. pont. max. ${ }^{5}$ trib. pot. XIIX imp. X/cos. VI p.p. / res $p$. Saeponensium / statuam triump(h)alem / ex d.d. dedit) nos ha permitido aclarar definitivamente este problema: Saepo, que pertenece, como dice Plinio, a las ciudades de la Beturia Céltica y al conventus Hispalensis, hay que situarla en los alrededores del cortijo de Vistalegre, y Vsaepo en la dehesa de la Fantasía, término de Cortes de la Frontera. Así, pues, las inscripciones CIL II 1339-1340 hay que leerlas como res p(ublica) Vsaeponensium y Plinio tenía razón una vez más: se trataba de dos ciudades distintas ubicadas precisamente donde este decía, la una al norte en el convento Hispalense y la otra mucho más al sur en el Gaditano.

Además de la mención pliniana, una ciudad de nombre Vgia aparece mencionada en la via Augusta, entre las mansiones de Hasta y Orippo, a 27 m.p. al norte de la primera y 24 m.p. al sur de la segunda (It. Ant., 410,1; Vicarell. I (Vgiam), II, III, IV (Vgiae); Roldán 1975: 275;
Sillières 1990: 311). También aparece mencionada en la Cosmografía del Anónimo de Rávena en una vía que unía Hispalis con Asido y que, al parecer, se continuaría hasta Baesippo a través del valle del Barbate (An. Rav. 317,4, Sillières 1990: 433-34). Tolomeo $(2.4,10)$ menciona, en territorio de los turdetanos, dos ciudades dife-

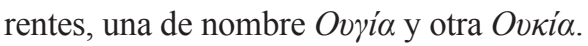

$\mathrm{Su}$ localización ha dividido a los estudiosos en dos grupos; uno, partidario de situarla en Las Cabezas de San Juan (Saavedra 1967: 105, Fita 1896: 366, Miller 1964: 156, Thouvenot 1940: 369-70 y 485, Collantes de Terán et al. 1943: II 1ss, Galsterer 1971: 22, 68, Galsterer-Kröll 1972: 109, aunque el matrimonio Galsterer se refiere a los Martienses qui antea Vgienses fuerunt, mencionados en una tabula patronatus encontrada en Mérida (AE 1952, 49), y que, en realidad, se trata de otra ciudad del conventus Cordubensis) y otro grupo en el yacimiento de Las Torres de Alocaz, situado en el km 590.5 de la carretera de Madrid a Cádiz, dentro del término municipal de Utrera (Mancheño 
y Olivares 1901-27: I 94, Sillières 1976: 58-59 y 1990: 311, González 1982b: 163). Romero de Torres (1934: 196) situaba Vgia, sin ningún fundamento, en la moderna Espera (Cádiz), donde se ha encontrado un fragmento de inscripción (EE VIII: 503) y Tovar (1974: 57) y Roldán (1975: 275) no se definen al respecto.

Nosotros creemos que efectivamente hay que localizar la Vgia pliniana en Las Torres de Alocaz, donde la inscripción de Rufinus (González 1982b: 153-154) nos confirma su adscripción a la tribu Galeria, propia de las fundaciones augusteas. Además, el reciente hallazgo de un ius iurandi pro salute Augusti, encontrado en las proximidades de Las Cabezas de San Juan, nos ha permitido ubicar en esta ciudad la antigua Conobaria y descartar la localización de Vgia en dicha localidad (González 1988: 13-14).

Sin embargo, la denominación dada por Plinio a esta ciudad: Vrgia Castrum Iulium, item Caesaris Salutariensis plantea no pocas dudas surgidas de la sorprendente duplicidad del cognomen, caso único en las titulaciones de ciudades y hace surgir la pregunta de si el adverbio item debe entenderse como introductor del segundo cognomen o de una segunda ciudad del mismo nombre: Vgia Castrum Iulium, item (Vgia) Caesaris Salutariensis; es decir, se trata de una ciudad con dos cognomina o mejor aún de dos ciudades diferentes, cada una con su propio cognomen.

Así, por ejemplo, Galsterer opina que se trata de dos comunidades diferentes, de emplazamiento desconocido (Galsterer 1971: 5, 68), e igual es el parecer de Hoyos, que defiende la diferente identidad de ambas, identificándolas con las comunidades citadas por Ptolomeo $(2.4,10)$ y las sitúa muy próximas entre sí, una en Las Cabezas de San Juan o en una zona próxima y la otra en algún lugar no muy alejado (Detlefsen 1870: 306, Hoyos 1979: 444-45).

Vemos, pues, cómo la identificación de esa segunda Vgia constituye el principal problema de los partidarios de mantener, de acuerdo al texto de Tolomeo, la existencia de dos ciudades con idéntico nombre, pero diferente titulación. Pensamos que tal vez nuestro fragmento puede contribuir a solucionar el problema y ello no solo por la inicial V- del nombre de la anónima ciudad existente en el despoblado de Gibalbín, sino también, lo que es más relevante, por el hecho de que ésta debía ser una ciudad Latio vetere, según el explícito testimonio del diploma militar del anónimo pretoriano.

En efecto, ya Plinio nos informa de que el status de Vgia Castrim Iulium, item Caesaris Salutariensis era el de una ciudad latina: Latinorum Laepia Regia, Carisa cognomine Aurelia, Vrgia cognominata Castrum
Iulium, item Caesaris Salutariensis, y aunque la mayoría de los estudiosos se inclina por considerarla fundación de César (Galsterer 1971: 5, 68, Galsterer-Kröll 1972: 109, Hoyos 1979: 44-45, Wiegels 1985: 62), no existe unanimidad en su condición de municipio iuris Latini (Corzo y Jiménez 1980: 32) o colonia latina (Tovar 1974: 57). Nosotros creemos que se trata de dos asentamientos coloniales de derecho latino (Henderson 1942: 7-10), transformados por Augusto, en una época desconocida, probablemente en el 13/15 a.C., en municipios, pues, según hemos ya establecido en diversos trabajos anteriores (González 1984: 27-30, 1986: 147-242), la lex Iulia municipalis fue aplicada en las provincias de Occidente por Augusto, lo que dificulta grandemente la existencia de tales municipios de derecho latino en época de César.

La autoría de Augusto viene confirmada por el hecho de que en la única inscripción en la que consta la tribu de Vgia, esta es la Galeria. Conocemos en un epígrafe encontrado en la provincia de Zamora (AE 1928, 180) a un soldado, M. Herenniu[s.-] f. Gal. Gallicus domo Vgia mil leg. X Gem [---], que parece muy probable que sea oriundo de una de estas dos Vgiae.

Otro dato incidental, pero de gran importancia, nos lo ofrece el siguiente epígrafe que en el siglo XVII se "encontraba delante de la puerta llamada de Sevilla, en la pared de las huertas de San Domingo" (CIL II 1305, González 1982: 103):

\section{L.FABIO.L.F.GAL.CORDO IIIIVIRo \\ 5 SALVTE.ET.VICTORIA.CAESARVM LOCVS.ET.INSCRIPTIO.D.D P|E|R.TABELLAM.DATA}

Cuya interpretación ha planteado algunas dudas sobre sobre el desarrollo de la abreviatura M.C. de la 1.3 y así, mientras algunos piensan en un m(unicipium) C(eretanum) (Esteve 1979: 103, Chic 1978: 40, Padilla 1989: 185-192), otros lo hacen en un m(unicipium) C(aesarinum) (Vittinghoff 1951: 104, Galsterer 1971: 20, Wiegels 1985: 17). Los primeros se apoyan en la existencia de una ciudad, de emplazamiento desconocido, conocida exclusivamente por sus monedas con leyenda CERIT (Vives 1924-26: 3.78), que se ha identificado con la actual Jerez de la Frontera, pues no hay inconveniente alguno en aceptar el desarrollo fonético de Ceret> Jerez (Pemán 1941: 96-97, Tovar 1973: 397400, Padilla 1989: 186-190), aunque no se haya podido 
confirmar la antigüedad de la moderna población con hallazgos arqueológicos del período romano, por lo que las inscripciones encontradas en Jerez o bien han sido reutilizadas como elementos constructivos, o bien han sido traídas de algún despoblado vecino, como es el caso de La Mesa de Asta, sin que haya constancia del hallazgo de ningún epígrafe en el casco urbano (González 1982: 101-119).

Los segundos se inclinan por pensar que el pedestal habría sido traído de la localidad de Medina Sidonia, antigua colonia Asido Caesarina, situada a casi 40 $\mathrm{km}$ de distancia, aunque no han tenido en cuenta que en la reutilización de elementos arqueológicos como materiales de construcción se produce desplazamiento de los mismos si se trata de un despoblado y su empleo en el lugar de su hallazgo, si la ciudad antigua ha continuado su existencia, como es el caso de Medina Sidonia, donde hay numerosos ejemplos del uso de materiales arqueológicos y epígrafes reutilizados en la construcción de edificios. Además, la evidencia de que los Caesares mencionados son Lucio y Gayo Césares, les obliga a suponer que ya era municipio el año 2 a.C. (Vittinghoff 1951: 104, Galsterer 1971: 20, Wiegels 1985: 17). Otros, en cambio, creen que o bien sería ya colonia antes del 27 a.C. (Thouvenot 1940: 190) o bien una colonia latina de César antes de recibir de Augusto el status de colonia civium Romanorum, según se deduce de la existencia en la colonia de IIIIviri y IIviri (Henderson 1942: 12-13, van Nostrand 1916: 115), opinión ésta que nos parece la más acertada (González 2011: 273-295). Vemos, pues, cómo la aceptación de esta posibilidad deja numerosas objeciones sin resolver.

Sea como sea, la expresión populus m(unicipii) $C$ (aesarini) o $C$ (eretani) resulta extraña a las normas jurídicas y no conocemos ningún ejemplo de la unión de populus y municipium. En efecto, en los documentos epigráficos las expresiones usuales son populus (CIL II $1089 ; 1185 ; 1364 ; 2100 ; 2162 ; 3270 ; 4514 ; 5515)$, senatus populusque (CIL II 1343; 5346; 3695), populus acompañado del étnico correspondiente (CIL II 1294), ordo et populus (CIL II $3221=6339)$, populus et incolae (CIL II 1286) y patria et populus (CIL II 6109). Por ello nos parecen arriesgadas las dos restituciones propuestas $m$ (unicipium) $C$ (aesarinum) y $C$ (eretanum), y consideramos más que probable que se trate de una mala lectura del original, toda vez que en esta posición del epígrafe esperaríamos un dativo HVIC y no la enigmática abreviatura M.C.

Una vez descartada la procedencia del epígrafe en cuestión, no deja de ser sugerente la posibilidad de que haya sido llevado a Jerez de la Frontera desde el despoblado de Gibalbín, perteneciente a su término municipal, dado que el personaje homenajeado L. Fabius Cordus estaba inscrito en la tribu Galeria, la que hemos supuesto propia de la anónima ciudad asentada en Gibalbín.

Una última cuestión, para la que desgraciadamente no tenemos una solución coherente, es cuál de las dos Vgiae tendría su asentamiento en Las Torres de Alocaz y cuál en Gibalbín. Tan sólo podemos deducir que la configuración del yacimiento de Gibalbín, un monte escarpado con una altura de $410 \mathrm{~m}$, encaja mejor con el significado de castrum como "lugar o asentamiento fortificado", por lo que nos inclinamos a situar aquí la Vgia Castrum Iulium y en Las Torres de Alocaz la Vgia Caesaris Salutariensis.

\section{BIBLIOGRAFÍA}

Albertini, E. (1923): Les divisions administratives de l'Espagne. París, E. de Boccard.

Chic, G. (1978): “Acerca de un ánfora con pepitas de uvas encontrada en la punta de la Nao (Cádiz)". Boletín del Museo de Cádiz 1: 40.

Chic, G. (1979-80): “Lacca”. Habis 10-11: 275-276.

Collantes de Terán, F., Hernández Díaz, J., Sancho Corbacho, A. (1943): Catálogo arqueológico y artístico de la provincia de Sevilla, 4 vols. Sevilla, Diputación Provincial de Sevilla.

Corzo, R. y Jiménez, A. (1980): “Organización territorial de la Bética". Archivo Español de Arqueología 53: 21-48.

Detlefsen, D. (1870): "Die Geographie der Provinz Baetica bei Plinius". Philologus 30: 303-04.

Esteve Guerrero, M. (1979): “Ceret y Asta Regia, dos ciudades distintas”, en M.A. García Paz (ed.), Miscelánea Arqueológica Jerezana: 103. Jerez, Centro de Estudios Históricos Jerezanos.

Fita, F. (1896): “Arcos de la Frontera. Excursión epigráfica”. Boletín de la Real Academía de la Historia $R A H$ 29: 366.

Fita, F. (1896): “Antiguos epígrafes de Tanger, Jerez y Arcos de la Frontera". Boletín de la Real Academía de la Historia RAH 29: 432-35.

Galsterer, H. (1971): Untersuchungen zum Römischen Städtewesen auf der Iberischen Halbinsel. Berlín, Walter de Gruyter.

Galsterer-Kroll, B. (1972): “Untersuchungen zu den Beinamen der Städte des Imperium Romanum". Epigraphische Studien 9: 1-102. 
García Iglesias, L. (1971): "La Beturia un problema geográfico de la Hispania Antiqua". Archivo Español de Arqueología 44: 86-108.

González, J. (1982): Inscripciones romanas de la provincia de Cádiz. Cádiz, Diputación Provincial de Cádiz.

González, J. (1982a): "Interrex y occisus est ab latronibus", I Congreso Andaluz de Estudios Clásicos: 223-227. Jaén, Diputación Provincial de Jaén.

González, J. (1982b): "Miscelánea epigráfica andaluza". Archivo Español de Arqueología 55: 153-172.

González, J. (1984): "Italica, municipium iuris Latini". Mélanges de la Casa de Velázquez XX: 17-43.

González, J. (1986): "The lex Irnitana: a new copy of the Flavian municipal law". Journal of Roman Studies $R S$ 76: 147-242.

González, J. (1987): “Trajano: Parthicus, trib. pot. XIIX, imp. X". Archivo Español de Arqueología 60: 237-250.

González, J. (1988): “The first oath pro salute Augusti found in Baetica". Zeitschrift für Papyrologie und Epigraphik 72: 113-127.

González, J. (1994): "Epigrafía jurídica de la Bética", en J. González (ed.), Roma y las provincias. Realidad administrativa e ideología imperial: 11-15. Madrid, Ediciones Clásicas.

González, J. (2011): “Asido quae Caesarina”, en J. González y J.C. Saquete (eds.), Colonias de César y Augusto en la Andalucía romana: 273-295. Roma, L'Erma di Bretschneider.

Henderson, M.I. (1942): "Iulius Caesar and Latium in Spain”. Journal of Roman Studies 32: 7-12.

Hoyos, B.D. (1979): "Pliny the Elder's titled Baetican towns: obscurities, errors and origins". Historia 28: 449.

Mancheño y Olivares, M. (1901-1927): Antigüedades del partido judicial de Arcos de la Frontera y pueblos que existieron en él. Arcos de la Frontera.

Miller, K.M. (1964): Itineraria romana. Römische Reisewege an der Hand der Tabula Peutingeriana dargestellt. Roma, L'Erma di Bretschneider.

Padilla Monge, A. (1989): "El epígrafe CIL II 1305". Habis 20: 185-192.
Pemán, C. (1941): El pasaje tartésico de Avieno. Madrid, Instituto Diego de Velázquez.

Pesserini, P. (1939): Les coorti pretorie. Roma, L'Erma di Bretschneider.

Raepsaet-Charlier, M. T. (1978): "Le lieu d'installation des vétérans Auxiliaires romains d'après les diplomes militaires". La Antiquité classique 47: 557-62.

Roldán, J.M. (1974): Hispania y el ejército romano. Contribución a la historia social de la España antigua. Salamanca, Universidad de Salamanca.

Roldán, J.M. (1975): Itineraria hispana. Valladolid, Universidad de Valladolid.

Romero de Torres, E. (1934): Catálogo Monumental de la provincia de Cádiz. Madrid, Ministerio de Instrucción Pública y Bellas Artes.

Saavedra, E. (1967): Discursos leídos ante la Real Academia de la Historia en la recepción de don Eduardo Saavedra. Madrid (1862, 1ª ed.), Real Academia de la Historia.

Sillières, P. (1976): "La vie Augusta de Cordove a Cadix". Mélanges de la Casa de Velázquez 12: 58-59.

Sillières, P. (1977): "Prospections le long de la via Augusta". Habis 8: 342-43.

Sillières, P. (1990): Les voies de communication de l'Hispanie meridionale. París, Diffusion De Boccard.

Thouvenot, R. (1940): Essai sur la province romaine de Bétique. París, Raymond.

Tovar, A. (1973): "Columela y el vino de Jerez", en Homenaje al profesor Carriazo. 3: 399-404. Sevilla, Universidad de Sevilla.

Tovar, A. (1974): IberischeLandeskunde. Zweiter Teil: Die Völkerund die Städte des antiken Hispanien, I: Baetica. Baden-Baden, Koemer.

Van Nostrand, J. (1916): Die Reorganisation Spaniens durch Augustus. Berkeley.

Vitinghoff, F. (1951): Römische Kolonisation und Bürgerrechtpolitik unter Caesar und Augustus. Mainz, Akademie der Wissenschaften und der Literatur.

Vives, A. (1924-26): La moneda hispánica. Madrid, Real Academia de la Historia.

Wiegels, R. (1985): Die Tribuschriften des Römischen Hispanien. Berlín, Walter de Gruyter. 